\title{
METÁSTASIS SUPRARRENAL METACRÓNICA EN 2 PACIENTES SOMETIDOS A NEFRECTOMÍA RADICAL POR CARCINOMA DE CÉLULAS RENALES PRIMARIO.
}

José Luis Rosales Leal, Francisco Rodríguez Herrera, Manuel Ortiz Gorráiz, Beatriz Honrubia Vílchez, Antonio Fernández Sánchez, Fernando Vázquez Alonso, Manrique Pascual Geler, Antonio Martínez Morcillo, Javier Vicente Prados, José Manuel Cózar Olmo, Eduardo Espejo Maldonado y Miguel Tallada Buñuel.

Servio de Urología. Hospital Universitario Virgen de las Nieves. Granada. España.

Resumen.- OBJETIVOS/MÉTODOS: Presentamos dos casos excepcionales de metástasis suprarrenales metacrónicas de carcinoma de células renales y revisamos la literatura existente. Valorados distintos aspectos como la frecuencia, etiopatogenia, diagnóstico y seguimiento de estos pacientes concluimos que estas metástasis son poco frecuentes y suelen presentarse tardías en su evolución en pacientes con CCR con bajo estadios anatomo-patológicos.

RESULTADOS/CONCLUSIONES: Revisado el tratamiento y constatando que no hay directrices en cuanto al manejo terapéutico de estos pacientes con metástasis proponemos la cirugía de la metástasis suprarrenal así como el tratamiento adyuvante con inmunoterapia.
José Luis Rosales Leal C/ Periodista José Fernández Martínez №4, Portal 1, 2A 18014 Granada. (España) irosal@fundacionhvn.org Trabajo recibido: 29 de mayo 2006
Palabras clave: Carcinoma de células renales. Glándula suprarrenal. Metástasis.

Summary.- OBJECTIVES: We report two exceptional cases of metachronous adrenal metastasis of renal cell carcinomas and perform a bibliographic review. After the evaluation of various features such as frequency, etiopathogenesis, diagnosis and follow-up of these patients we conclude that these metastases are rare, and they usually appear late in the evolution of patients with low stage renal cell carcinoma.

RESULTS/CONCLUSIONS: Once reviewed the treatment and checked the absence of guidelines for the therapeutic management of these patients we propose surgery for the adrenal metastasis as well as adjuvant treatment with immunotherapy.

Keywords: Renal cell carcinoma. Adrenal gland. Metastasis.

\section{INTRODUCCIÓN}

En la actualidad, la mayoría de los tumores renales se diagnostican en fases precoces de la enfermedad, como masas incidentales o tumores de bajo estadio. Sin embargo, las metástasis pueden aparecer en el momento del diagnóstico (sincrónicas) o pueden ocurrir a lo largo de muchos años de seguimiento (metacrónicas), en diversas e inesperadas localizaciones. La mayoría de las metástasis de carcinoma de células renales (CCR) se localizan en 
pulmón, hígado, hueso y ganglios linfáticos. La afectación suprarrenal (SR) maligna metacrónica es excepcional. Presentamos dos casos de metástasis SR y revisamos la literatura.

\section{CASOS CLÍNICOS}

\section{CASO 1}

Mujer de 67 años, con antecedentes personales de HTA, TBC pulmonar con paquipleuritis residual, hipotiroidismo primario en tratamiento sustitutivo, suprarrenalectomía izquierda en 1992 por síndrome de Cushing, intervenida de cataratas y polipectomía colónica. Se diagnosticó incidentalmente de cáncer renal mediante estudio ecográfico y TAC y fue sometida a nefrectomía radical derecha en 1997. El diagnóstico anatomo-patológico fue compatible con adenocarcinoma renal de células claras, grado nuclear 2 de Furhman, en polo inferior, de $6 \mathrm{~cm}$ de diámetro ( $\mathrm{PT}$ I bNOMO).

Ha sido revisada cada seis meses durante los 3 primeros años, y posteriormente cada año, mediante analítica, Rx de tórax y pruebas de imagen (TAC abdomino-pélvico y ecografía abdominal), según protocolo. Presentó una masa SR (Figura 1) en el seguimiento a los 95 meses, por lo que fue valorada por Servicio de Endocrinología que descartó funcionalidad. El estudio de extensión fue negativo. Se realizó una suprarrenalectomía derecha por vía abierta con informe anatomo-patológico de metástasis metacrónica de CCR, con patrón de células claras. Comenzó con tratamiento corticoideo sustitutivo.

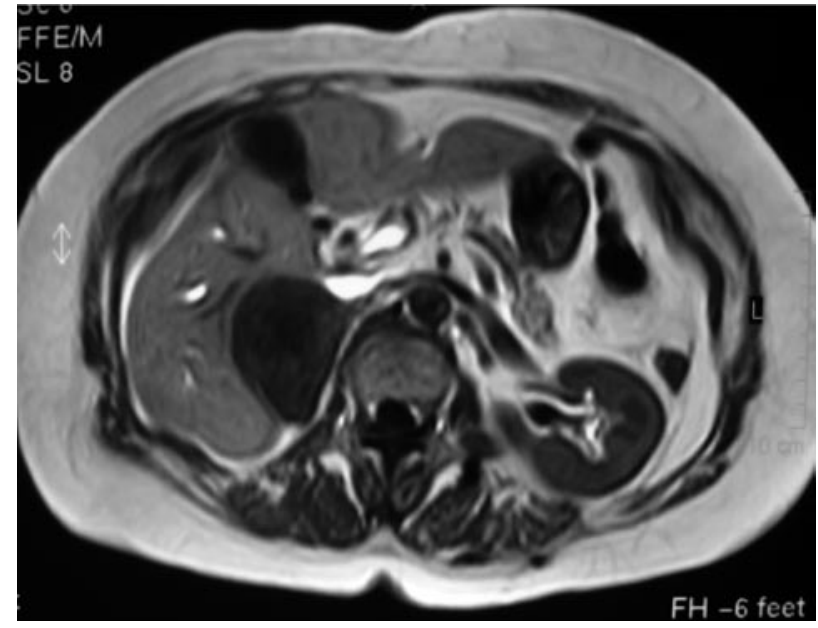

FIGURA 1. Caso I RMN Mx SR derecha.

Inició inmunoterapia adyuvante con Interleuquina 2 + interferón alfa según protocolo del Servicio (Tabla ( A), que suspendió durante el primer ciclo por deterioro de la función renal. A los 3 meses de la intervención se evidenció la presencia de metástasis pulmonares múltiples de pequeño tamaño, mediante TAC torácico. Constatada la enfermedad metastásica pulmonar se reinició inmunoterapia al 50\% bajo el esquema terapéutico según protocolo en pacientes metastáticos (Tabla I B) precisando doblar las dosis de corticoides en todos los ciclos. A los 8 meses de seguimiento desde el diagnóstico y tratamiento de la masa SR permanece estable clínicamente, con estabilización de sus metástasis pulmonares.

\section{TABLA I A. PROTOCOLO DE TRATAMIENTO ADYUVANTE CON INMUNOTERAPIA EN EL CCR LOCALMENTE} AVANZADO.

Inicio: 3ciclos de 21 días/mes IL-2 (9mUI/día) + INF- $\alpha$ (3mUI/días 3ํy 5을 le la semana), vía subcutánea

Mantenimiento: $12-18$ ciclos de 7 días/mes IL-2 $(9 \mathrm{mUl} /$ día $)+\left(\mathrm{NF}-\alpha\left(3 \mathrm{mUI} / \mathrm{d} 3^{\circ}\right.\right.$ y $5^{\circ}$ de la sem.) vía sc Evaluación: Analítica mensual; TAC abd.-pélvico y Rx tórax trimestral; $G$. ósea si dolor/elevación FA

TABLA I B. PROTOCOLO DE TRATAMIENTO CON INMUNOTERAPIA EN EL CCR METASTÁSICO.

Inicio: 6 ciclos de 21 días/mes IL-2 $(9 \mathrm{mUI} /$ día $)+\mathrm{INF}-\alpha\left(3 \mathrm{mUI} /\right.$ días $3^{\circ}$ y $5^{\circ}$ de la semana), vía subcutánea Mantenimiento: ciclos de 7 días/mes IL-2 (9mUI/día) + INF- $\alpha\left(3 \mathrm{mUI} / \mathrm{d} 3^{\circ}\right.$ y $5^{\circ}$ de la sem.) vía sc Evaluación: Analítica mensual; TAC abd.-pélvico y Rx tórax trimestral; G. ósea si dolor/elevación FA 


\section{CASO 2}

Varón de 53 años, con antecedentes personales de HTA, obesidad mórbida, TEP, TVP, timoma estadio II Masaoka, tratado en 1992 mediante timectomía y radioterapia adyuvante (45Gy) en remisión. Presentó dolor en fosa renal izquierda diagnosticándose de cáncer renal mediante estudio de ECO y TAC. Fue sometido a nefrectomía radical y suprarrenalectomía izquierdas en 1999 con diagnóstico anatomo-patológico de adenocarcinoma renal de células mixtas (claras y granulares), grado nuclear 2 de Furhman, de polo superior, de $8 \mathrm{~cm}$. de diámetro (pT2NOMO).

Ha seguido el mismo protocolo de revisión. Se sometió a suprarrenalectomía derecha por presentar metástasis SR metacrónica derecha a los 70 meses de seguimiento (Figura 2), con informe anatomopatológico para metástasis de CCR con patrón mixto (células claras y mixtas). Considerado como enfermo de alto riesgo con posible evolución metastásica, ha inició protocolo de inmunoterapia adyuvante (Tabla (A) con buena tolerancia tras el primer ciclo.

A los 9 meses de seguimiento permanece estable clínicamente, presentando en TAC torácico de control engrosamiento pleural de aspecto nodular, situado en la región postero-basal derecha, pudiendo corresponder a metástasis, recomendándose punción aspiración con aguja fina para su filiación.

Por otra parte también se ha identificado una adenopatía latero-cervical izquierda de unos $2 \mathrm{~cm}$ clínica y ecográfica, pendiente de su exéresis y estudio anatomopatológico, ya que la PAAF realizada no fue diagnóstica.

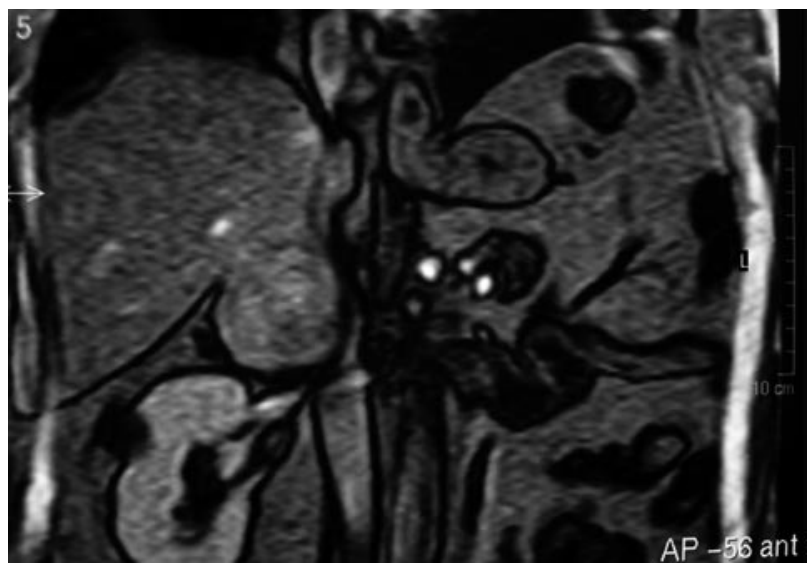

FIGURA 2. Caso2 RMN Mx SR.

\section{DISCUSIÓN}

El CCR representa el 3\% de las enfermedades malignas del adulto. Ocupa el tercer lugar en las neoplasias urológicas, por detrás del cáncer de próstata y vesical. En Estados Unidos la tasa de incidencia ajustada por edad de este proceso para hombres blancos, mujeres blancas y para negros es de $9,6,11,1$ y 4,9 por 100.000 habitantes-año respectivamente (1). En Europa la incidencia estimada en 2004 es de 3,89 y 2,26 por 100.000 habitantes-año para hombres y mujeres (2). Dependiendo de las series, aproximadamente el 60 y el $70 \%$ de los pacientes presentan enfermedad localizada o localmente avanzada potencialmente curable con tratamiento quirúrgico (3). Sin embargo, las tasas de recurrencia son elevadas (35-65\%) para pacientes con tumores localmente agresivos, dependiendo del estadio patológico, grado nuclear de Furhman y ECOG-PS (4). El resto de los pacientes presentan metástasis múltiples a distancia, siendo las más frecuentes en pulmón, ganglios linfáticos, hígado o hueso (5). El comportamiento del CCR es impredecible, hasta el $50 \%$ de los pacientes sometidos a una nefrectomía radical inicialmente curativa, desarrollará enfermedad metastásica metacrónica durante su evolución en el tiempo, por lo que precisan seguimientos a largo plazo (6).

La afectación metastásica SR del CCR es poco frecuente (7). De hecho, la presencia de signos y síntomas clínicos de insuficiencia adrenal son poco prevalentes. La frecuencia de afectación SR metastásica como hallazgo anatomopatológico en series de autopsias en pacientes con CCR se ha situado entre 10 y el $19 \%(10,9)$. La metástasis SR suele ser más frecuente en la glándula ipsilateral. Saitoh (8), en más de 400 casos, encontró un 17\% de afectación ipsilateral y un $11 \%$ de afectación contralateral, con afectación exclusiva de la glándula SR contralateral del 2,5\%. En una serie de 610 pacientes observaron una incidencia de afectación SR ipsilateral sincrónica del 3.4\% (21/610) y contralateral del $1.1 \%(7 / 610)$, de las que sólo 4 fueron metacrónicas (0.6\%) (9). En otra revisión publicada la frecuencia de metástasis metacrónica fue del $1,4 \%(5 / 350)(10)$.

La afectación suprarrenal ipsilateral puede ocurrir por diseminación hematógena de células aisladas o por trombosis tumoral a través de la vena renal, vena adrenal, venas colaterales de la grasa perinéfrica y periféricas de la glándula adrenal. La extensión directa a través de la cápsula renal puede ocurrir en tumores de gran tamaño o de polo superior. Aunque las metástasis contralaterales son menos frecuentes que la afectación suprarrenal ipsilateral, se ha sugeri- 
do que la glándula adrenal constituye un medio ambiente favorable para el desarrollo de metástasis del CCR en la diseminación hematógena (11).

En una cohorte retrospectiva de pacientes intervenidos de CCR en nuestro centro se diagnosticaron 5 casos con metástasis SR. Entre 1988 y 2005 se intervinieron 238 pacientes diagnosticados de CCR. La edad media (mediana, rango) de los pacientes intervenidos fue de 60,4 años $(62,18-89)$. Presentaron metástasis en diversas localizaciones en el control evolutivo el $11,7 \%(28 / 238)$, con un seguimiento medio (desviación típica, rango) de 36,5 meses $(33,8 ; 3-139)$. Se realizó nefrectomía radical con suprarrenalectomía en el 31,9\% (76/238) con afectación SR en un 3,9\% $(3 / 76)$ de los pacientes (1 bilateral, 1 ipsilateral, 1 contralateral). Se han descrito dos casos de metástasis SR metacrónicas que constituyen el $0,8 \%$ del total de la serie $(2 / 238)$, similar a la serie de Von Knobloch (9).

Se han comunicado diagnósticos de metástasis SR metacrónicas hasta 23 años después de una nefrectomía (12). Este desfase cronológico se puede explicar debido a que algunas metástasis pueden ser de muy bajo crecimiento, sobretodo si son de bajo grado, y por fallo en el uso de estudios de imagen de rutina en algunos casos (13).

Las metástasis SR suelen ser anatómicamente y funcionalmente silentes. Se desconoce el sustrato biológico que determina la afectación contralateral aislada.

TABLA II. RESULTADO AP DE PACIENTES SOMETIDOS A NEFRECTOMIIA RADICAL Y SUPRARRENALESCTOMÍA EN NUESTRO SERVICIO (1988-2005).

\begin{tabular}{|c|c|c|c|}
\hline $\begin{array}{c}\text { Estadio AP } \\
\text { (TNM 2002) }\end{array}$ & № pacientes & $\%$ & $\begin{array}{c}\text { № SR } \\
\text { patológicas }\end{array}$ \\
\hline pT1a & 5 & 6.6 & 0 \\
\hline pT1b & 12 & 15.7 & 0 \\
\hline PT2 & 26 & 34.3 & 1 \\
\hline pT3a & 18 & 23.7 & 0 \\
\hline pT3b & 10 & 13.1 & 1 \\
\hline pT3c & 0 & 0 & 0 \\
\hline PT4 & 5 & 6.6 & 1 \\
\hline Total & 76 & 100 & 3 \\
\hline
\end{tabular}

Respecto al tratamiento primario del CCR, ha sido controvertido a lo largo de los años la necesidad - no de adrenalectomía ipsilateral simultánea con la nefrectomía. Diversos estudios han concluido que este procedimiento no conlleva asociado una mejoría del pronóstico de la enfermedad salvo en determinadas circunstancias (14-16). Se han establecido como factores de riesgo de afectación suprarrenal estadios tumorales elevados, localización en polo superior, multifocalidad, trombosis de la vena renal así como sospecha de afectación mediante imagen por TAC (valor predictivo negativo del $99,4 \%)(16,17)$. En nuestra serie de 238 pacientes intervenidos por CCR en el periodo 1988-2005, se indicó nefrectomía radical con suprarrenalectomía ipsilateral por afectación del hemirriñón superior en el 31,9\% (76/238), con 3 casos de afectación SR, que constituyó un 3,9\% (3/76). En la Tabla II se correlaciona el estadio tumoral anatomopatológico con la afectación SR.

Debido al reducido número de pacientes con este tipo de metástasis a largo plazo hace difícil llegar a conclusiones sobre su pronóstico y manejo. La supervivencia de los pacientes con enfermedad metastásica avanzada sin tratamiento tienen un pronóstico pobre, pero éste difiere en aquellos pacientes con lesiones solitarias en las que es posible su extirpación quirúrgica completa, consiguiendo una supervivencia prolongada con tasas del 23 al $35 \%$ a los 5 años. $(9,10,18)$. El pronóstico es más favorable en aquellos pacientes diagnosticados con un intervalo de tiempo mayor desde la nefrectomía (19). Laul y cols. (13) revisaron 56 casos de metástasis SR contralaterales publicados hasta el 2001, en los que se realizó adrenalectomía como tratamiento. 24 lesiones fueron sincrónicas y 32 metacrónicas. El rango de edad fue de 43 a 82 años, el mayor tiempo comunicado de aparición de la metástasis SR después de la nefrectomía fue de 23 años, con un seguimiento de 0,3 a 14,3 años 27 permanecían sin evidencias de enfermedad y 17 pacientes murieron por CCR metastático. Actualmente se considera la vía laparoscópica como vía de abordaje inicial en el tratamiento quirúrgico de la afectación SR (20). Aunque empíricamente los pacientes sometidos a tratamiento quirúrgico de la metástasis del CCR podrían beneficiarse de tratamiento adyuvante inmunoterápico, no existen estudios que aborden esta cuestión $(21,22,25)$.

Nosotros consideramos a este grupo pacientes de riesgo para el desarrollo de enfermedad metastásica, como ha sucedido en estos dos casos aislados. Así que proponemos la realización de tratamiento quirúrgico citorreductor (exéresis de la metástasis única cuando sea factible), asociado a pautas de inmunoterapia adyuvante, aunque en la literatura científica no se haya concluido hasta la fecha el esquema terapéutico más eficaz. 
Por otra parte, los diversos esquemas quimioterápicos o radioterápicos empleados no han permitido controlar la enfermedad localmente avanzada o diseminada. Hasta este momento, para la enfermedad metastásica se han desarrollado múltiples regimenes de inmunoterapia basados en el uso del interferón alfa y la interleuquina 2, en monoterapia o en asociaciones siendo el Interferón $\alpha$ el que aporta mayores resultados en la literatura.

En la actualidad se están desarrollando ensayos clínicos fase III que tratan de evaluar el uso sinérgico de la IL-2 asociado a Interferón $\alpha$, que han conseguido tasas de respuesta (remisión parcial o completa) entre el 15 y el $25 \%$. Por otra parte se han identificado factores pronósticos para predecir el beneficio del tratamiento con citoquinas, y ayudar al médico a seleccionar a los pacientes. Estos factores incluyen la anemia, elevación de la $\mathrm{LDH}$, elevación del calcio, ausencia del tratamiento quirúrgico previo, un bajo Karnofsky/ECOG performal status, número, tamaño y localización de las metástasis.

Estudios recientes han examinado la eficacia de agentes antiangiogénicos, como el bevacizumab, un inhibidor del factor de crecimiento endotelial vascular (VEGF), así como otra serie de fármacos con diferentes mecanismos de acción basados en el desarrollo del conocimiento molecular en la patogénesis de esta enfermedad, de cuya utilidad podrá disponerse en breve (23-26).

En conclusión, podemos decir que las metástasis SR de CCR metacrónicas son poco frecuentes y suelen presentarse tardías en su evolución en pacientes con CCR con bajo estadios anatomo-patológicos. $\mathrm{Se}$ desconocen los mecanismos de escape tumoral a tan largo plazo para el desarrollo de enfermedad metastásica. No disponemos de series amplias previas que establezcan directrices en cuanto al manejo terapéutico adecuado de estos pacientes con metástasis SR. En casos seleccionados de lesiones únicas pueden ser subsidiarias de exéresis quirúrgica. Aunque no hay evidencias científicas que apoyen el tratamiento adyuvante con inmunoterapia en CCR localmente avanzado no metastático en los ensayos clínicos publicados, todavía no se han realizado este tipo de estudios de adyuvancia en pacientes con metástasis única tratados con cirugía.

Consideramos a este tipo de pacientes de alto riesgo para el desarrollo de enfermedad metastásica múltiple, por lo que recomendaríamos tratamiento empírico adyuvante con inmunoterapia (IL2 + INFa). Creemos necesario el desarrollo de ensayos clínicos que demuestren su beneficio.

\section{BIBLIOGRAFÍA y LECTURAS} RECOMENDADAS ( ${ }^{*}$ lectura de interés $y^{* *}$ lectura fundamental)

1. CHOW, W.H.; DEVESA, S.S.; WARREN, J.L. y cols.: "Rising incidence of renal cell cancer in the United States". JAMA, 281: 1628, 1999.

2. BOYLE, P.; FERLAY, J.: "Cancer incidence and mortality in Europe, 2004”. Ann. Oncol., 16: 481, 2005.

*3. ORTIZ GORRAIZ, M.; VICENTE PRADOS, F.J.; ROSALES LEAL, J.L. y cols.: "Valoración de los factores pronósticos de la supervivencia en una serie de 202 pacientes intervenidos por carcinoma de células renales". Actas Urol. Esp., 29: 179, 2005.

**4. LAM, J.S.; LEPPERT, J.T.; BELLDEGRUN, A,.S. y cols.: "Adjuvant therapy of renal cell carcinoma: patient selection and therapeutic options". BJU Int., 96: 483, 2005.

5. KOZLOWSKI, J.M.: "Management of distant solitary recurrence in the patient with renal cancer: contralateral kidney and other sites". Urol. Clin. North Am., 21: 601, 1994.

6. SANCHEZ ZALABARDO, D.; AROCENA GGARCIA-TAPIA, J.; REGOJO BALBOA, J.M. y cols.: "Factores pronóstico en carcinoma renal pT3”. Actas Urol. Esp., 27: 26, 2003.

*7. CRUZ GUERRA, N.A.; LINARES QUEVEDO, A.; CLEMENTE RAMOS, L. y cols.: "Metástasis adrenal metacronica solitaria contralateral de un carcinoma renal primario: aportacion de un nuevo caso". Arch. Esp. Urol., 54: 825, 2001.

8. SAITOH, H.; NAKAYAMA, M.; NAKAMURA, K. y cols.: "Distant mestastasis of adenocarcinoma in nephrectomized cases". J. Urol., 127: 1092, 1982.

9. VON KNOBLOCH; HEGELE, A.; KÄLBLE, T. y cols.: "Management of contralateral adrenal metastasis from renal cell carcinoma: possibility of inferior vena cava tumour thrombus". Scand. J. Urol. Nephrol., 34: 109, 2000.

**10. KESSLER, O.J.; MUKAMEL, E.; WEINSTEIN, R. y cols.: "Metachronous renal cell carcinoma metastasis to the contralateral adrenal gland". Urology, 51: 539, 1998.

11. SAGALOWSKY, A.I.; MOLBERG, K.: "Solitary metastasis of renal cell carcinoma to the contralateral adrenal gland 22 years after nephrectomy". Urology, 54: 162, 1999.

12. MESOROLLE, B.; MIGNON, F.; TRAVAGLI, J.P. y cols.: "Late presentation of solitary contralateral adrenal metastasis of renal cell carcinoma". Eur. Radiol., 7: 557, 1997.

*13. LAU, W.K.; ZINCKE, H.; LOHSE, C.M. y cols.: "Contralateral adrenal metastasis of renal cell carcinoma: treatment, outcome, and review". BJU Int., 91: 775, 2003. 
*14. YOKOYAMA, H.; TANAKA, M.: "Incidence of adrenal involvement and assessing adrenal function in patients with renal cell carcinoma: is ipsilateral adrenalectomy indispensable during radical nephrectomy?". BJU Int., 95: 526, 2005.

15. PAUL, R.; MORDHORST, J.; LEYH, H. y cols.: "Incidence and outcome of patients with adrenal metastases of renal cell cancer". Urology, 57: 878, 2001.

16. TSUI, K.H.; SHVARTS, O.; BARBARIC, Z. y cols.: "Is adrenalectomy a necessary component of radical nephrectomy? UCLA experience with 511 radical nephrectomies". J. Urol., 163: 437, 2000.

17. MOUDOUNI, S.M.; EN-NIA, I.; PATARD, J.J. y cols.: "Real indications for adrenalectomy in renal cell carcinoma". Scand. J. Urol. Nephrol., 36: 273, 2002.

**18. SENGUPTA, S.; LEIBOVICH, B.C.; BLUTE, M.L. y cols.: "Surgery for metastatic renal cell cancer". World J. Urol., 23: 155, 2005.

19. DRUCKER, B.J.: "Renal cell carcinoma: current status and future prospects". Cancer Treat. Rev., 31: 536, 2005.

20. ELASHRY, O.M.; CLAYMAN, R.V.; SOBLE, J.J. y cols.: "Laparoscopic adrenalectomy for solitary metachronous contralateral adrenal metastasis from renal cell carcinoma". J. Urol., 157: 1217, 1997.

21. JEON, S.H.; CHANG, S.G.; KIM, J.I.: "The role of adjuvant immunotherapy after radical nephrectomy and prognostic factors in pT3N0M0 renal cell carcinoma". Anticancer Res., 19: 5593, 1999.

*22. LAM, J.S.; LEPPERT, J.T.; BELLDEGRUN, A.S. y cols.: "Adjuvant therapy of renal cell carcinoma: patient selection and therapeutic options". BJU Int., 96: 483, 2005.

*23. CÓZAR, J.M.; TALLADA, M.; ESPEJO, E. y cols.: "Estado actual de la inmunoterapia en el cáncer renal diseminado". Rev. Urol., 3: 62, 2002.

24. LAM, J.S.; LEPPERT, J.T.; BELLDEGRUN, A.S. y cols.: "Novel approaches in the therapy of metastatic renal cell carcinoma". World J. Urol., 23: 202, 2005.

**25. COPPIN, C.; PORZSOLT, F.; AWA, A. y cols.: "Immunotherapy for advanced renal cell cancer. The Cochrane Database of Systematic Reviews 2004, Issue 3". Art. No.: CD001425. DOI: 10.1002/14651858.CD001425.pub2.

*26. COHEN, H.T.; McGOVERN, F.J.: "Renal cell carcinoma”. N. Engl. J. Med., 353: 2477, 2005. 